\title{
Can vitamin D supplementation help control inflammation in inflammatory bowel disease beyond its classical role in bone health?
}

\author{
Sung Wook Hwang \\ Department of Gastroenterology and Inflammatory Bowel Disease Center, Asan Medical Center, University of Ulsan College of Medicine, Seoul, \\ Korea
}
Article: The effect of vitamin D administration on inflammatory markers in patients with inflam- matory bowel disease (Intest Res 2019;17:210-217)

Inflammatory bowel disease (IBD) is a chronic immune-mediated disorder with a complicated pathogenesis, and with increasing prevalence in both Western and Eastern countries, it has emerged as a public health challenge worldwide. ${ }^{1}$ Patients with IBD are known to be at risk for several nutritional deficiencies. ${ }^{2}$ Regarding vitamin D and calcium homeostasis patients with IBD have an increased risk of osteopenia and osteoporosis, defined by the umbrella term metabolic bone disease. ${ }^{3}$ Several factors, such as malabsorption of calcium and/ or vitamin D due to disease flare or surgery, diminished food intake, and medication, can interfere with bone metabolism, while other non-disease-related factors, such as low sunlight exposure, insufficient physical activity, and smoking, may also contribute to vitamin D deficiency in IBD. ${ }^{4}$ Vitamin D deficiency appears to be prevalent in IBD patients, with a reported rate ranging from $16 \%$ to $95 \%$, and it is more frequent in patients with CD than in those with UC., Vitamin D deficiency is traditionally known to cause impaired calcium absorption, negative calcium balance, excessive bone resorption, and con-

Received March 28, 2019. Accepted March 31, 2019.

Correspondence to Sung Wook Hwang, Department of Gastroenterology and Inflammatory Bowel Disease Center, Asan Medical Center, University of Ulsan College of Medicine, 88 Olympic-ro 43-gil, Songpa-gu, Seoul 05505, Korea. Tel: +82-2-3010-3195, Fax: +82-2-476-0824, E-mail: hsw903@ gmail.com

ORCID Sung Wook Hwang (https://orcid.org/0000-0002-6981-7575) sequently metabolic bone disease. Moreover, vitamin D has its own functions in the immunomodulation of both innate and adaptive immunity and influences the gut microbiome beyond the classical role in bone health. ${ }^{3}$

In the current issue of Intestinal Research, Jun et al. ${ }^{5}$ investigated the association of vitamin D status with CRP level and the partial Mayo score in patients with IBD. In addition, whether vitamin D supplementation could influence the biochemical marker and disease activity was explored. The authors enrolled 88 patients with $\mathrm{CD}$ and 178 patients with UC, and serum 25-hydroxyvitamin $\mathrm{D}_{3}[25(\mathrm{OH}) \mathrm{D}]$ levels were measured to evaluate vitamin $\mathrm{D}$ status. In patients with $\mathrm{CD}$, a negative correlation was found between 25(OH)D and CRP (Spearman's rho $=-0.259 ; 95 \% \mathrm{CI},-0.427$ to -0.078 ), while no significant correlation of $25(\mathrm{OH}) \mathrm{D}$ with CRP or partial Mayo score was reported in patients with UC. Following vitamin D supplementation for 6 months, the 25(OH)D levels significantly increased in both CD and UC patients ( $11.08 \pm 3.63$ to $22.69 \pm 6.11 \mathrm{ng} / \mathrm{mL}$ in CD and $11.45 \pm 4.10$ to $24.20 \pm 6.61 \mathrm{ng} / \mathrm{mL}$ in UC). However, this study failed to show a significant effect of vitamin D supplementation on CRP reduction and partial Mayo score between the vitamin D normalized and non-normalized groups in $\mathrm{CD}$ and UC. Serum 25(OH)D is the major circulating form of vitamin D, and it is usually used to define vitamin D status in research and clinical practice. ${ }^{4}$ Although the threshold for 
the deficiency level has not been well established in the literature, serum 25(OH)D levels between 20 and $30 \mathrm{ng} / \mathrm{mL}$ are considered insufficient and levels $\leq 20 \mathrm{ng} / \mathrm{mL}$ are considered deficient. ${ }^{3}$ In the study by Jun et al., ${ }^{5}$ an increase in vitamin D levels after supplementation was shown, but it still did not achieve sufficient levels.

Vitamin D deficiency seems to be inversely linked to disease activity, clinical relapse, frequent hospitalization, and poor quality of life in patients with IBD, although previous studies have reported conflicting data., ${ }^{3,6}$ The study by Jun et al., ${ }^{5}$ revealed an inverse correlation between serum 25(OH)D levels and CRP in CD, and a recent study from Korea also revealed a significant inverse correlation of vitamin D levels with disease activity in CD patients. ${ }^{7}$ Nonetheless, most of the previous studies were retrospective or cross-sectional in design, thus, they did not answer the chicken or the egg casualty dilemma. Recently, prospective studies have shown an association of low vitamin D levels with clinical relapse, steroid and biologics use, hospitalization, and surgery. ${ }^{4}$ In terms of response to biologics, several studies have explored the relationship between 25(OH)D levels and response to anti-TNF inhibitors, ${ }^{4,6}$ in which vitamin D levels appear to influence response and durability of anti-TNF therapy. Taken together, these results support the relevance of maintaining adequate levels of vitamin D in patients with IBD in order to improve disease course and response to conventional therapeutics. ${ }^{3,4,6}$

The Western guidelines such as the European Crohn's and Colitis Organisation consensus and the American College of Gastroenterology clinical guideline recommended the assessment of vitamin D levels and supplementation with calcium and vitamin D for the prevention of metabolic bone disease. ${ }^{8,9}$ The European Society for Parenteral and Enteral Nutrition guideline also recommended the supplementation of calcium and vitamin $\mathrm{D}$ with the rationale that vitamin $\mathrm{D}$ deficiency is associated with an increased risk of surgery, hospitalization and Clostridium difficile infection in patients with CD and UC in addition to the prevention of osteoporosis. ${ }^{10}$ There have been a few randomized controlled trials that support the relevance of the guidelines beyond the classical role of vitamin $\mathrm{D}$ in calcium metabolism and bone health, in which vitamin D supplementation reduced CRP level, disease activity, and clinical relapse in IBD. ${ }^{4}$ However, the available studies have confounders or limitations; thus, this uncertain area remains to be investigated. The study by Jun et al., ${ }^{5}$ in which the authors unfortunately failed to show the effect of vitamin D supplementation, also has similar limitations such as small sample size, inadequate dose, and possibility of poor compliance. There is no consensus about adequate dose and duration of vitamin D supplementation and follow-up timing of vitamin D measurement to date. Supplementing all patients with the same dose of vitamin D might be inadequate to maintain therapeutic threshold. ${ }^{4}$ To clarify these unsolved questions, a well-designed randomized controlled study focusing on the effect of vitamin D supplementation on outcome in IBD is necessary.

\section{FINANCIAL SUPPORT}

The authors received no financial support for the research, authorship, and/or publication of this article.

\section{CONFLICT OF INTEREST}

No potential conflict of interest relevant to this article was reported.

\section{AUTHOR CONTRIBUTION}

Writing and approval of final manuscript: Hwang SW.

\section{REFERENCES}

1. Yen HH, Weng MT, Tung CC, et al. Epidemiological trend in inflammatory bowel disease in Taiwan from 2001 to 2015: a nationwide populationbased study. Intest Res 2019;17:54-62.

2. Yoon SM. Micronutrient deficiencies in inflammatory bowel disease: trivial or crucial? Intest Res 2016;14:109-110.

3. Ananthakrishnan AN. Vitamin D and inflammatory bowel disease. Gastroenterol Hepatol (N Y) 2016;12:513-515.

4. Nielsen OH, Rejnmark L, Moss AC. Role of vitamin D in the natural history of inflammatory bowel disease. J Crohns Colitis 2018;12:742-752.

5. Jun JC, Yoon H, Choi YJ, et al. The effect of vitamin D administration on inflammatory markers in patients with inflammatory bowel disease. Intest Res 2019;17:210-217.

6. Gubatan J, Moss AC. Vitamin D in inflammatory bowel disease: more than just a supplement. Curr Opin Gastroenterol 2018;34:217-225.

7. Ko KH, Kim YS, Lee BK, et al. Vitamin D deficiency is associated with disease activity in patients with Crohn's disease. Intest Res 2019;17:70-77.

8. Magro F, Gionchetti P, Eliakim R, et al. Third European evidencebased consensus on diagnosis and management of ulcerative 
colitis. Part 1: definitions, diagnosis, extra-intestinal manifestations, pregnancy, cancer surveillance, surgery, and ileo-anal pouch disorders. J Crohns Colitis 2017;11:649-670.

9. Farraye FA, Melmed GY, Lichtenstein GR, Kane SV. ACG clinical guideline: preventive care in inflammatory bowel disease.
Am J Gastroenterol 2017;112:241-258.

10. Forbes A, Escher J, Hébuterne X, et al. ESPEN guideline: clinical nutrition in inflammatory bowel disease. Clin Nutr 2017; 36:321-347. 